University of Warwick institutional repository: http://go.warwick.ac.uk/wrap This paper is made available online in accordance with publisher policies. Please scroll down to view the document itself. Please refer to the repository record for this item and our policy information available from the repository home page for further information.

To see the final version of this paper please visit the publisher's website. Access to the published version may require a subscription.

Author(s): Roger Fenn, Colin Rourke and Brian Sanderson

Article Title: James bundles

Year of publication: 2004

Link to published

version: http://dx.doi.org/10.1112/S0024611504014674

Publisher statement: None 


\section{JAMES BUNDLES}

\section{ROGER FENN, COLIN ROURKE AND BRIAN SANDERSON}

This paper is concerned with $\square$-sets, pronounced 'square sets', which are cubical sets without degeneracies. These arise naturally in a number of settings and can be used to classify links of codimension 2. Here we shall describe some of the intrinsic geometry of a $\square$-set and, in particular, we shall define an infinite family of associated $\square$-sets, which we call James complexes. These define a sequence of mock bundles with base the given $\square$-set, the James bundles, which have a strong connection with classical James-Hopf invariants. They define natural characteristic classes for a $\square$-set and lead to associated generalised cohomology theories with geometric interpretation similar to that for Mahowald orientation $[\mathbf{1 9}, \mathbf{2 3}]$.

In an earlier paper [10] we explored the abstract connections between $\square$-sets and categories through the concept of a trunk which can be regarded both as a generalisation of the (cubical) nerve of a category and as a $\square$-set. We defined a classifying space ( $\square$-set) for a rack (the rack space) and used the trunk formalism to establish connections with classical classifying spaces (for categories, groups and crossed complexes). In [12] we shall apply the results of [10] and this paper by showing that the rack space is a classifying space for smooth links (of codimension 2) with the first James bundle as classifying bundle. Further in [13] we shall give a classification of classical links using racks and the canonical class in $\pi_{2}$ of the rack space determined by the link diagram.

This paper formed part of our January 1996 preprint 'James bundles and applications' [11], which also contains a preliminary version of [12]. For historical background see [8].

Further results on $\square$-sets are to be found in $[\mathbf{1}]$ and $[\mathbf{2}]$. In $[\mathbf{1}]$ it is shown that the natural map $C \rightarrow T$ of a $\square$-set to the trivial $\square$-set induces a homotopy equivalence $|C| \simeq \Omega\left(S^{2}\right) \times R$ in the case when $C$ admits degeneracies and $R$ is the realisation using degeneracies. In particular, the singular $\square$-set $\mathrm{S}(X)$ of a space $X$ has the weak homotopy type $\Omega\left(S^{2}\right) \times X$. This result is given an alternative proof in $[\mathbf{2}]$ in terms of the natural interpretation of maps into $C$ as labelled diagrams, which comes from using transversality; see [12]. However it is not the case that all naturally occurring infinite $\square$-sets split a copy of $\Omega\left(S^{2}\right)$ up to homotopy type. For example, rack spaces do not in general split in this way (see the computations of homotopy type of rack spaces in $[\mathbf{1 2}, \mathbf{2 5}])$. Moreover, a general $\square$-set can have an arbitrary weak homotopy type (Corollary 1.3).

This paper is organised as follows. In $\S 1$ we define $\square$-sets, give examples and explain the connection with $\Delta$-sets. In $\S 2$ we define the James complexes and James bundles of a $\square$-set and in $\S 3$ we give the connection with the classical James-Hopf invariants. In $\S 4$ we consider the classes in stable cohomotopy and in cohomology determined by the James bundles and show how these classes are

Received 1 February 2003; revised 5 September 2003.

2000 Mathematics Subject Classification 55N22, 55P44 (primary), 57R15, 57R20, 57R90 (secondary). 
pulled back from Steifel-Whitney and Wu classes of $B O$. In $\S 5$ we use the map to $B O$ to define the concept of a $\square$-set orientation for a manifold. This leads to a generalised (co)-homology theory which has a geometric interpretation close to that for Mahowald orientation given in [23].

\section{Basic definitions and examples}

A $\square$-set $C=\left\{C_{n} ; \partial_{i}^{\epsilon}\right\}$ consists of a collection of sets $C_{n}$, one for each natural number $n \geqslant 0$, and functions $\partial_{i}^{\epsilon}: C_{n} \rightarrow C_{n-1}$, for $1 \leqslant i \leqslant n$ and $\epsilon \in\{0,1\}$, called first order face maps, satisfying the following face relations:

$$
\partial_{j-1}^{\eta} \partial_{i}^{\epsilon}=\partial_{i}^{\epsilon} \partial_{j}^{\eta}, \quad \text { for } 1 \leqslant i<j \leqslant n \text { and } \epsilon, \eta \in\{0,1\} \text {. }
$$

There is a notion of a $\square$-map $f: C \rightarrow D$ between $\square$-sets, namely a collection of maps $f_{n}: C_{n} \rightarrow D_{n}$ for $n=0,1, \ldots$, which commute with the face maps, that is, $f_{n-1} \partial_{i}^{\epsilon}=\partial_{i}^{\epsilon} f_{n}$.

Note that a $\square$-set differs from a cubical set, which has a similar definition but also has degeneracy maps; for more detail on the connections between cubical and $\square$-sets, see $[\mathbf{1 0}]$.

There is also the notion of a $\square$-space. This comprises a collection $\left\{X_{n}\right\}$ of topological spaces and first order face maps (continuous maps) $\partial_{i}^{\epsilon}: X_{n} \rightarrow X_{n-1}$ for $1 \leqslant i \leqslant n$ and $\epsilon \in\{0,1\}$ satisfying the same face relations as above.

We shall give presently alternative definitions for $\square$-sets and maps based on cubes. First we need to establish some terminology.

\section{The category}

The $n$-cube $I^{n}$ is the subset $[0,1]^{n}$ of $\mathbb{R}^{n}$.

A $p$-face of $I^{n}$ is a subset defined by choosing $n-p$ coordinates and setting some of these equal to 0 and the rest to 1 . In particular there are $2 n$ faces of dimension $n-1$ determined by setting $x_{i}=\epsilon$ where $i \in\{1,2, \ldots, n\}$ and $\epsilon \in\{0,1\}$.

A 0 -face is called a vertex and corresponds to a point of the form $\left(\epsilon_{1}, \epsilon_{2}, \ldots, \epsilon_{n}\right)$ where $\epsilon_{i}=0$ or 1 for $i \in\{1,2, \ldots, n\}$. The 1 -faces are called edges and the 2 -faces are called squares.

Let $p \leqslant n$ and let $J$ be a $p$-face of $I^{n}$. Then there is a canonical face map $\lambda: I^{p} \rightarrow I^{n}$, with $\lambda\left(I^{p}\right)=J$, given in coordinate form by preserving the order of the coordinates $\left(x_{1}, \ldots, x_{p}\right)$ and inserting $n-p$ constant coordinates which are either 0 or 1 . If $\lambda$ inserts only 0 s we call it a front face map, and if it inserts only 1s we call it a back face map. Notice that any face map has a unique front-back decomposition as $\lambda \mu$, say, where $\lambda$ is a front face map and $\mu$ is a back face map. There is also a unique back-front decomposition. There are $2 n$ face maps defined by the $(n-1)$-faces which are denoted $\delta_{i}^{\epsilon}: I^{n-1} \rightarrow I^{n}$, and given by

$$
\delta_{i}^{\epsilon}\left(x_{1}, x_{2}, \ldots, x_{n-1}\right)=\left(x_{1}, \ldots, x_{i-1}, \epsilon, x_{i}, \ldots, x_{n-1}\right), \quad \text { with } \epsilon \in\{0,1\} .
$$

The following relations hold:

$$
\delta_{i}^{\epsilon} \delta_{j-1}^{\omega}=\delta_{j}^{\omega} \delta_{i}^{\epsilon}, \quad \text { for } 1 \leqslant i<j \leqslant n \text { and } \epsilon, \omega \in\{0,1\} .
$$

Definition. The category $\square$ is the category whose objects are the $n$-cubes $I^{n}$ for $n=0,1, \ldots$ and whose morphisms are the face maps. 


\section{$\square$-Sets and their realisations}

We now give the second equivalent definitions of $\square$-sets and maps.

A $\square$-set is a functor $C$ : $\square^{\text {op }} \rightarrow$ Sets where $\square^{\text {op }}$ is the opposite category of $\square$ and Sets denotes the category of sets.

A $\square$-map between $\square$-sets is a natural transformation.

We write $C_{n}$ for $C\left(I^{n}\right), \lambda^{*}$ for $C(\lambda)$ and we write $\partial_{i}^{\epsilon}$ for $C\left(\delta_{i}^{\epsilon}\right)=\left(\delta_{i}^{\epsilon}\right)^{*}$.

The equivalence of the two definitions of $\square$-sets and maps follows from the formulæ for the composition of face maps given in equation (1.1) above.

The realisation $|C|$ of a $\square$-set $C$ is given by making the identifications $\left(\lambda^{*} x, t\right) \sim(x, \lambda t)$ in the disjoint union $\coprod_{n \geqslant 0} C_{n} \times I^{n}$.

We shall call 0-cells, 1-cells and 2-cells of $|C|$ vertices, edges and squares, respectively, and this is consistent with the previous use for faces of $I^{n}$, since $I^{n}$ determines a $\square$-set with cells corresponding to faces, whose realisation can be identified in a natural way with $I^{n}$.

Notice that $|C|$ is a CW complex with one $n$-cell for each element of $C_{n}$ and that each $n$-cell has a canonical characteristic map from the $n$-cube.

However, not every CW complex with cubical characteristic maps comes from a $\square$-set, even if the cells are glued by isometries of faces. In $|C|$, where $C$ is a $\square$-set, cells are glued by face maps, in other words by canonical isometries of faces.

There is a similar alternative definition of a $\square$-space namely a functor $X$ : $\square^{\mathrm{op}} \rightarrow$ Top (where Top denotes the category of topological spaces and continuous maps) and the realisation $|X|$ given by the same formula as above. Note that a $\square$-set is effectively the same as a $\square$-space in which all spaces have the discrete topology.

\section{Connection with $\Delta$-sets}

The concept of a $\Delta$-set is similar to the concept of a $\square$-set but is based on simplexes rather than cubes. A $\Delta$-set can have an arbitrary (weak) homotopy type; see $[\mathbf{2 2}]$ where basic material on $\Delta$-sets can be found. The definition of a $\Delta$-set can be obtained from the first definition of a $\square$-set (at the start of the section) by forgetting the $\epsilon \mathrm{s}$ and $\eta \mathrm{s}$ and allowing $i=0$.

A $\square$-set can be subdivided to form a $\Delta$-set by coning from centres. More precisely suppose that $C$ is a $\square$-set. Define the $\Delta$-subdivision, $\operatorname{Sd}_{\Delta}(C)$, as follows. A $k$-simplex of $\operatorname{Sd}_{\Delta}(C)$ is a $(k+1)$-tuple $\left(c, \lambda_{1}, \ldots, \lambda_{k}\right)$ where $c \in C_{n}$, for some $n$, $\lambda_{i}: I^{n_{i-1}} \rightarrow I^{n_{i}}$ is a face map, $1 \leqslant i \leqslant k$, and $n_{0}<n_{1}<\ldots<n_{k}=n$. The face maps $\partial_{i}$, for $0 \leqslant i \leqslant k$, are given by

$$
\partial_{i}\left(c, \lambda_{1}, \ldots, \lambda_{k}\right)= \begin{cases}\left(c, \lambda_{2}, \ldots, \lambda_{k}\right) & \text { for } i=0, \\ \left(c, \lambda_{1}, \ldots, \lambda_{i-1}, \lambda_{i+1} \lambda_{i}, \lambda_{i+2}, \ldots, \lambda_{k}\right) & \text { for } 0<i<k \\ \left(\lambda_{k}^{*} c, \lambda_{1}, \ldots, \lambda_{k-1}\right) & \text { for } i=k\end{cases}
$$

Conversely given a $\Delta$-set $X$ we can define a $\square$-subdivision, $\operatorname{Sd}_{\square}(X)$ of $X$ by projecting simplexes from the origin. More precisely, let

$$
\Delta^{n}=\left\{x \in \mathbb{R}^{n+1} \mid x_{i} \geqslant 0, \sum_{i} x_{i}=1\right\}
$$

denote the standard $n$-simplex. Let $\rho_{n}: \Delta^{n} \rightarrow \partial I^{n+1}$ be the radial projection from $0 \in \mathbb{R}^{n+1}$. Then $\rho_{n}\left(\Delta^{n}\right)$ is the union of the back $n$-faces of $I^{n+1}$. A picture of the image of $\Delta^{2}$ under this projection (seen from the origin) is given in Figure 1, and 
it can be seen that $\Delta^{2}$ has been subdivided into three squares. A similar projection subdivides an $n$-simplex into $n+1 n$-cubes and this process defines the required $\square$-subdivision.

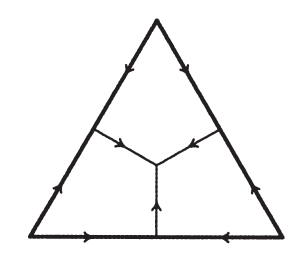

Figure 1.

For detailed formulæ, let $B(k, n)$ denote the set of back face maps $\lambda: I^{k} \rightarrow I^{n}$. If $\lambda: I^{k} \rightarrow I^{n}$ is a front face map let $r(\lambda): \Delta^{k-1} \rightarrow \Delta^{n-1}$ denote its restriction. Define the $\square$-subdivision, $\operatorname{Sd}_{\square}(X)$ as follows. Set

$$
\operatorname{Sd}_{\square}(X)_{k}=\coprod_{n>k} X_{n-1} \times B(k, n) .
$$

Suppose given $x \in X_{n-1}, \lambda \in B(k, n)$ and a face map $\mu: I^{s} \rightarrow I^{k}$. Let $\lambda^{\prime} \mu^{\prime}$ be the front-back decomposition of $\lambda \mu$. Define $\mu^{*}(x, \lambda)=\left(r\left(\lambda^{\prime}\right)^{*} x, \mu^{\prime}\right)$.

1.2. Proposition. (1) For any $\Delta$-set $X$ the spaces $|X|$ and $\left|\operatorname{Sd}_{\square}(X)\right|$ are homeomorphic.

(2) For any $\square$-set $C$ the spaces $|C|$ and $\left|\operatorname{Sd}_{\Delta}(C)\right|$ are homeomorphic.

Proof. The homeomorphism $\left|\operatorname{Sd}_{\square}(X)\right| \rightarrow|X|$ is induced by

$$
(x, \lambda, t) \in X_{n-1} \times B(k, n) \times I^{k} \longmapsto\left(x, \rho^{-1}(\lambda(t))\right) \in X_{n-1} \times \Delta_{n-1} .
$$

Let $\widehat{c}_{m}=\left(\frac{1}{2}, \ldots, \frac{1}{2}\right) \in I^{m}$. The homeomorphism $\left|\operatorname{Sd}_{\Delta}(C)\right| \rightarrow|C|$ is induced by

$$
\begin{aligned}
\left(\left(c, \lambda_{1}, \ldots, \lambda_{k}\right), \mathbf{t}\right) \in & \operatorname{Sd}_{\Delta}(C)_{k} \times \Delta_{k} \\
& \longmapsto\left(c, t_{k} \widehat{c}_{n}+\sum_{0 \leqslant i \leqslant k-1} t_{i} \lambda_{k} \ldots \lambda_{i+1} \widehat{c}_{n_{i}}\right) \in X_{n} \times I^{n},
\end{aligned}
$$

where $\mathbf{t}=\left(t_{1}, \ldots, t_{k}\right) \in \Delta_{k}$. Further details are left to the reader.

1.3. Corollary. Any space has the weak homotopy type of some $\square$-set.

Notation. We shall often omit the mod signs and use the notation $C$ both for the $\square$-set $C$ and its realisation $|C|$. We shall use the full notation whenever there is any possibility of confusion.

\subsection{EXAMPLES OF $\square$-SETS.}

1. The singular complex. Let $X$ be a topological space. The singular complex of $X$ is the $\square$-set denoted $\mathrm{S}(X)$ and defined by:

$\mathrm{S}(X)_{n}=\left\{f: I^{n} \rightarrow X\right\}$, the set of continuous maps, and

$\lambda^{*}(f)=f \circ \lambda$, where $\lambda$ is a face map. 
If $g: X \rightarrow Y$ is a continuous map then $\mathrm{S}(g): \mathrm{S}(X) \rightarrow \mathrm{S}(Y)$ is the $\square$-map defined by $\mathrm{S}(g)(f)=f \circ g$. Thus $\mathrm{S}(\cdot)$ is a functor from Top to the category of $\square$-sets and maps.

An important special case is the singular complex of a point. This has exactly one cell in each dimension and we shall call it the trivial $\square$-set and denote it $T$.

2. The nerve of a category. (For full details on this example, see [10].) Let $I_{\text {cat }}^{n}$ denote the category whose objects are the vertices of $I^{n}$. Morphisms are generated by the oriented edges of $I^{n}$ and subject to relations given by taking squares to be commutative diagrams. Each face map $\lambda: I^{q} \rightarrow I^{n}$ determines a functor $\lambda_{\text {cat }}: I_{\text {cat }}^{q} \rightarrow I_{\text {cat }}^{n}$. Let $\mathcal{C}$ be a category; the nerve of $\mathcal{C}$, denoted $\mathcal{N C}$, is the $\square$-set defined in analogy to the singular set:

$\mathcal{N C}_{n}=\left\{f: I_{\text {cat }}^{n} \rightarrow \mathcal{C}\right\}$, the set of functors and

$\lambda^{*}(f)=f \circ \lambda_{\text {cat }}$, where $\lambda$ is a face map.

Given a functor $g: \mathcal{C} \rightarrow \mathcal{D}$ between categories, we have a $\square$-map $\mathcal{N}(g): \mathcal{N C} \rightarrow \mathcal{N D}$, given by $\mathcal{N}(g)(f)=f \circ g$.

3. The rack space. A rack is a set $R$ with a binary operation written $a^{b}$ such that $a \mapsto a^{b}$ is a bijection for all $b \in R$ and such that the rack identity

$$
a^{b c}=a^{c b^{c}}
$$

holds for all $a, b, c \in R$. (Here we use the conventions for order of operations derived from exponentiation in arithmetic. Thus $a^{b c}$ means $\left(a^{b}\right)^{c}$ and $a^{c b^{c}}$ means $a^{c\left(b^{c}\right)}$.)

For example if $a, b \in G$ are two members of a group then conjugation, given by $a^{b}:=b^{-1} a b$, defines a rack structure on $G$. If $a, b \in \mathbb{Z}$ are two integers then $a^{b}:=a+1$ defines a rack structure on $\mathbb{Z}$ which does not come from conjugation in a group. Another example of a rack associated to a group is the core of a group [5, 16] defined by $a^{b}:=b a^{-1} b$. For more examples of racks see $[\mathbf{9}]$.

If $R$ is a rack, the rack space is the $\square$-set denoted $B R$ and defined by:

$B R_{n}=R^{n}$ (the $n$-fold cartesian product of $R$ with itself);

$\partial_{i}^{0}\left(x_{1}, \ldots, x_{n}\right)=\left(x_{1}, \ldots, x_{i-1}, x_{i+1}, \ldots, x_{n}\right)$;

$\partial_{i}^{1}\left(x_{1}, \ldots, x_{n}\right)=\left(\left(x_{1}\right)^{x_{i}}, \ldots,\left(x_{i-1}\right)^{x_{i}}, x_{i+1}, \ldots, x_{n}\right)$ for $1 \leqslant i \leqslant n$.

More geometrically, we can think of $B R$ as the $\square$-set with one vertex, with (oriented) edges labelled by rack elements and with squares which can be pictured as part of a link diagram with arcs labelled by $a, b$ and $a^{b}$ (Figure 2).

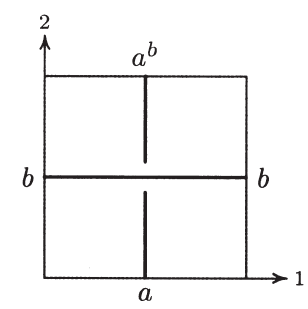

FiguRE 2. Diagram of a typical 2-cell of the rack space.

The higher dimensional cubes are determined by the squares in a way which was made precise in $[\mathbf{1 0}]$ and which we now summarise. 
A trunk $T$ comprises a set $T_{0}$ of vertices, a set $T_{1}$ of directed edges (between vertices) and a set $T_{2}$ of oriented squares of edges (the preferred squares). Thus a trunk is essentially a cubical set truncated at dimension 2 ; but notice that a square is fully determined by its faces (edges). A trunk $T$ has a nerve $\mathcal{N} T$ which is a $\square$-set defined in a similar way to the nerve of a category (above). The nerve has vertices, edges and squares in bijection with those of $T$ and higher cubes determined by the 2-skeleton as follows. Let $I_{\text {trunk }}^{n}$ be the trunk determined by the 2-skeleton of $I^{n}$; then $\mathcal{N} T_{n}=\left\{f: I_{\text {trunk }}^{n} \rightarrow T\right\}$, the set of trunk maps, with face maps given, as usual, by composition. This construction is fully compatible with the nerve of a category; indeed a category determines a trunk with preferred squares being the commuting squares; the two nerves are then identical.

Now a rack $R$ determines the trunk $T(R)$ with one vertex, edges the set $R$ and preferred squares of the type pictured above. The rack spaces $B R$ and the nerve of the trunk $\mathcal{N} T(R)$ coincide. In $[\mathbf{1 0}]$ the trunk formalism is used to establish connections between the rack space and other classifying spaces.

Notice that the rack space of the rack with one element has precisely one cube in each dimension. This is another description of the trivial $\square$-set.

4. The knot space. Let $\mathcal{I}_{n}$, for $n=0,1, \ldots$, denote the set of isotopy classes of smooth immersions (singular knots) $K$ of $S^{1}$ in $S^{3}$ with the following properties:

(1) the immersion $K$ is an embedding except at $n$ double points none of which involve the base-point of $S^{1}$;

(2) at each double point the two tangents are linearly independent.

Then $\mathcal{I}=\left\{\mathcal{I}_{n}\right\}$ can be made into a $\square$-set as follows. Let $K$ be a singular knot defining $[K] \in \mathcal{I}_{n}$. Number the double points of $K$ in order round the knot starting from the base-point and define $\partial_{i}^{1}([K])=\left[K_{i}^{+}\right]$where $K_{i}^{+}$is obtained from $K$ by unwrapping the $i$ th double point to give a positive crossing and $\partial_{i}^{0}([K])$ is similarly defined using a negative crossing. The face relations given at the start of the section are easily verified.

\section{Digression on Vassiliev invariants}

The knot space can be used to interpret Vassiliev invariants. A Vassiliev function $V=\left\{V_{n}\right\}$ on a $\square$-set $X$ with values in an abelian group $G$ is a sequence of functions $V_{n}: X_{n} \rightarrow G$ for each $n=0,1, \ldots$ satisfying the Vassiliev identity

$$
V_{n}(x)=V_{n-1}\left(\partial_{i}^{1} x\right)-V_{n-1}\left(\partial_{i}^{0} x\right) \quad \text { for } i=1,2, \ldots, n \text { and } x \in X_{n} .
$$

If $X$ is the knot space $\mathcal{I}$, a Vassiliev function is the usual notion of Vassiliev invariant [24]. The $\square$-set setting can be used to interpret the fundamental integration problem for Vassiliev invariants (see Bar-Natan [3]) in terms of cohomological obstructions. For more details see $[\mathbf{1 1}, \S 7]$.

\section{James complexes and bundles}

In this section we define, for any $\square$-set $C$, an infinite family of associated $\square$-sets $J^{n}(C)$, with $n \geqslant 0$, called the James complexes of $C$. These define mock bundles $\zeta^{n} / C$ which we call James bundles of $C$. 


\section{Projections}

An $(n+k, k)$-projection is a function $\lambda: I^{n+k} \rightarrow I^{k}$ of the form

$$
\lambda:\left(x_{1}, x_{2}, \ldots, x_{n+k}\right) \longmapsto\left(x_{i_{1}}, x_{i_{2}}, \ldots, x_{i_{k}}\right),
$$

where $1 \leqslant i_{1}<i_{2}<\ldots<i_{k} \leqslant n+k$.

Let $P_{k}^{n+k}$ denote the set of $(n+k, k)$-projections. Note that $P_{k}^{n+k}$ is a set of size

$$
\left(\begin{array}{c}
n+k \\
k
\end{array}\right)
$$

Let $\lambda \in P_{k}^{n+k}$ and let $\mu: I^{l} \rightarrow I^{k}$, with $l \leqslant k$, be a face map. The projection $\mu^{\sharp}(\lambda) \in P_{l}^{n+l}$ and the face map $\mu_{\lambda}: I^{n+l} \rightarrow I^{n+k}$ are defined uniquely by the following pull-back diagram:

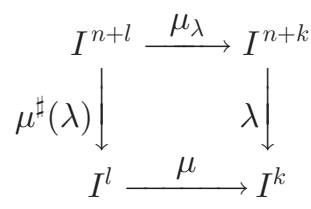

Definition. Let $C$ be a $\square$-set. The $n$th associated James complex of $C$, denoted $J^{n}(C)$, is defined as follows. The $n$-cells are given by

$$
J^{n}(C)_{k}=C_{n+k} \times P_{k}^{n+k}
$$

and face maps by

$$
\mu^{*}(x, \lambda)=\left(\mu_{\lambda}^{*}(x), \mu^{\sharp}(\lambda)\right)
$$

where $\mu: I^{l} \rightarrow I^{k}$, with $l \leqslant k$, is a face map.

Notation. Let $\lambda \in P_{k}^{n+k}$ and $c \in C_{n+k}$. Then we shall use the notation $c_{\lambda}$ for the $k$-cube $(c, \lambda) \in J^{n}(C)$. When necessary, we shall use the full notation $\left(\lambda_{1}, \ldots, \lambda_{n}\right)$ for the projection $\lambda$ (given by formula (2.1)) where $\lambda_{1}<\lambda_{2}<\ldots<\lambda_{n}$ and $\left\{\lambda_{1}, \ldots, \lambda_{n}\right\}=\{1, \ldots, k+n\}-\left\{i_{1}, \ldots, i_{k}\right\}$. In other words, we index cubes of $J^{n}$ by the $n$ directions (in order) which are collapsed by the defining projection.

\section{Picture for James complexes}

We think of $J^{n}(C)$ as comprising all the codimension $n$ central subcubes of cubes of $C$. For example a 3-cube $c$ of $C$ gives rise to the three 2-cubes of $J^{1}(C)$ which are illustrated in Figure 3.

In Figure 3 we have used full notation for projections. Thus for example, $c_{(2)}$ corresponds to the projection $\left(x_{1}, x_{2}, x_{3}\right) \mapsto\left(x_{1}, x_{3}\right)$ ( $x_{2}$ being collapsed).

This picture can be made more precise by considering the section $s_{\lambda}: I^{k} \rightarrow I^{n+k}$ of $\lambda$ given by

$$
s_{\lambda}\left(x_{1}, x_{2}, \ldots, x_{k}\right)=\left(\frac{1}{2}, \ldots, \frac{1}{2}, x_{1}, \frac{1}{2}, \ldots, \frac{1}{2}, x_{2}, \frac{1}{2}, \ldots, \frac{1}{2}, x_{k}, \frac{1}{2}, \ldots\right)
$$

where the non-constant coordinates are in places $i_{1}, i_{2}, \ldots, i_{k}$ and $\lambda$ is given by $(2.1)$.

For example, in the picture the image of $s_{\lambda}$ where $\lambda\left(x_{1}, x_{2}, x_{3}\right) \mapsto\left(x_{1}, x_{3}\right)$ is the 2-cube labelled $c_{(2)}$. 


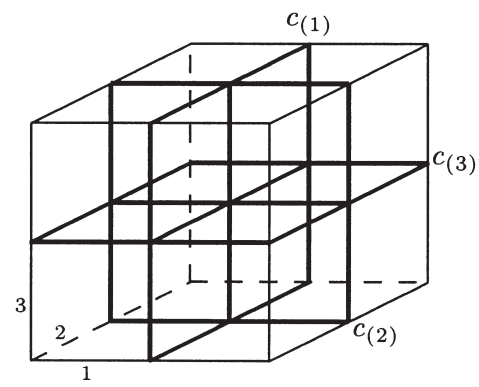

FIGURE 3 .

Now the commuting diagram (2.2) which defines the face maps implies that the $s_{\lambda}$ are compatible with faces and hence they fit together to define a map

$$
p_{n}:\left|J^{n}(C)\right| \rightarrow|C| \text { given by } p_{n}\left[c_{\lambda}, t\right]=\left[c, s_{\lambda}(t)\right] .
$$

\section{James bundles}

We now observe that $p_{n}:\left|J^{n}(C)\right| \rightarrow|C|$ is a mock bundle projection. We shall need to define a mock bundle with base a $\square$-set. Mock bundles with base a cell complex are defined (in a PL setting) in $[\mathbf{6}]$. In $[\mathbf{1 2}]$ we extend the concept to define a smooth mock bundle with base a smooth CW complex. For the purposes of this paper, we shall not need to pay attention to smooth technicalities and shall only need a minor extension of the treatment in $[\mathbf{6}]$.

Definition (Mock bundle over a $\square$-set). Let $C$ be a $\square$-set. A mock bundle $\xi$ over $C$ of codimension $q$ (denoted $\xi^{q} / C$ ) comprises a total space $E_{\xi}$ and a projection $p_{\xi}: E_{\xi} \rightarrow|C|$ with the following property. ${ }^{\dagger}$

Let $c$ be an $n$-cell of $|C|$ with characteristic map $\chi_{c}: I^{n} \rightarrow|C|$. Then there are a manifold (with corners) $B_{c}$ of dimension $n-q$ called the block over $c$ and a proper map $p_{c}: B_{c} \rightarrow I^{n}$ and a map $b_{c}: B_{c} \rightarrow E_{\xi}$ such that the following diagram is a pull-back:

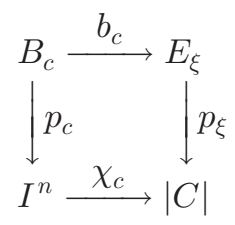

Key EXAmple (James bundles). Consider the projection

$$
p_{n}:\left|J^{n}(C)\right| \longrightarrow|C|,
$$

where $J^{n}(C)$ is the $n$th associated James complex of the $\square$-set $C$.

\footnotetext{
${ }^{\dagger}$ Note that the notation used here for dimension of a mock bundle, namely that $q$ is codimension, is the negative of that used in $[\mathbf{6}]$ where $\xi^{q} / C$ meant a mock bundle of fibre dimension $q$, that is, codimension $-q$. The notation used here is consistent with the usual convention for cohomology.
} 
If we choose a particular $(n+k)$-cell $\sigma$ of $C$ then the pull back of $p_{n}$ over $I^{n+k}$ (by the characteristic map for $\sigma$ ) is a $k$-manifold (in fact it is the $\left(\begin{array}{c}n+k \\ k\end{array}\right)$ copies of $I^{k}$ corresponding to the elements of $\left.P_{k}^{n+k}\right)$. Therefore $p_{n}$ is the projection of a mock bundle of codimension $n$, which we shall call the $n$th James bundle of $C$ denoted $\zeta^{n}(C)$.

\section{Embedding the James bundles in $|C| \times \mathbb{R}$}

Let $C$ be a $\square$-set. The James bundles can be embedded in $|C| \times \mathbb{R}$. This is done by ordering the cubes of $J^{n}(C)$ over a particular cube of $C$ and lifting in that order. Recall that the $k$-cubes of $J^{n}(C)$ lying over a $(k+n)$-cube are indexed by projections $\lambda=\left(i_{1}, \ldots, i_{n}\right) \in P_{n}^{n+k}$. These may be ordered lexicographically. The lexicographic order is compatible with face maps and can be used to define the required embedding by induction on dimension of cells of $C$ as follows.

Suppose inductively that the embedding has been defined over cells of $C$ of dimension at most $k+n-1$.

Consider a $(k+n)$-cube $c \in C$ with characteristic map $\chi_{c}: I^{k+n} \rightarrow|C|$. Pulling the embedding back (where it is already defined) over $\chi_{c}$ gives an embedding of $\zeta^{n}\left(\partial I^{k+n}\right)$ in $\partial I^{k+n} \times \mathbb{R}$. Now embed the centres of the $k$-cubes of $J^{n}\left(I^{k+n}\right)$ at $\left(\frac{1}{2}, \ldots, \frac{1}{2}\right) \times r_{\lambda}$ where $r_{\lambda}$ are real numbers for $\lambda \in P_{n}^{n+k}$ chosen to increase strictly corresponding to the lexicographic order on $P_{n}^{n+k}$.

Now embed each $k$-cube of $J^{n}\left(I^{k+n}\right)$ as the cone on its (already embedded) boundary. The result may be smoothed if desired and then pushed forward to $|C| \times \mathbb{R}$ using $\chi_{c} \times$ id.

In the next section we shall give precise smooth formulæ for this embedding using a bump function.

The embedding is in fact framed. This can be seen as follows. Each $k$-cube $p_{n}(c, \lambda)$, where $\lambda=\left(i_{1}, \ldots, i_{n}\right)$, of $J^{n}\left(I^{k+n}\right)$ is framed in $I^{k+n}$ by the $n$ vectors parallel to directions $i_{1}, \ldots, i_{n}$. These lift to parallel vectors in $I^{n+k} \times \mathbb{R}$ and the framing is completed by the vector parallel to the positive $\mathbb{R}$ direction (vertically up). This framing is compatible with faces and defines a framing of $\zeta^{n}(C)$ in $|C| \times \mathbb{R}$. The formulæ that we shall give in the next section also give formulæ for the framing.

For the special case $n=1$ the map of $\zeta^{n}(C)$ to $\mathbb{R}$ can be simply described: the centre of $c_{(k)}$ is mapped to $k$. This determines a map, linear on simplexes of $\mathrm{Sd}_{\Delta} \zeta^{1}(C)$, to $\mathbb{R}$. It follows that the centre of $\partial_{i}^{\epsilon} c_{(k)}$ is mapped to $k$ if $i \geqslant k$ and to $k-1$ if $i<k$.

In Figure 3 we illustrated $J^{1}(C)$ for a 3-cube $c \in C$. The embeddings in $|C| \times \mathbb{R}$ (before smoothing) above each of the three 2-cubes are illustrated in Figure 4.

\section{James-Hopf invariants}

In this section we shall show that James complexes are strongly connected with classical James-Hopf invariants; indeed they define (generalised) James-Hopf invariants for any $\square$-set $C$ and this explains our choice of terminology. We do this by looking carefully at James' original construction $[\mathbf{1 4}, \mathbf{1 5}]$ and in the process we give precise formulæ for the framed embedding of the James bundles in $|C| \times \mathbb{R}$. 


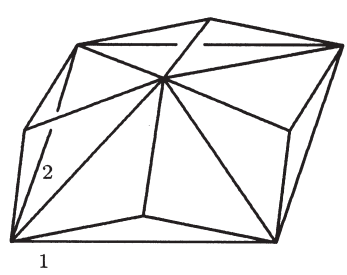

$c_{(3)}$

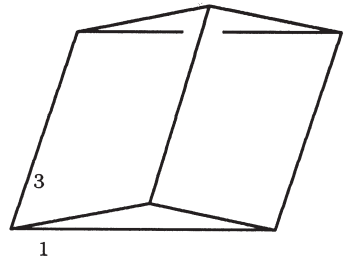

$c_{(2)}$

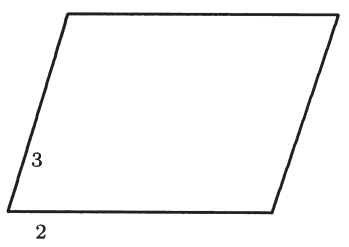

$c_{(1)}$

FiguRe 4.

\section{The James construction}

Definition (Free topological monoid). Let $X$ be a topological space based at $*$. The free topological monoid on $X$, denoted $X_{\infty}$ comprises all words $x_{1} \ldots x_{m}$, where $x_{i} \in X$ and $m \geqslant 0$ with the identifications given by regarding $*$ as a unit; that is,

$$
x_{1} \ldots x_{i-1} * x_{i+1} \ldots x_{m} \sim x_{1} \ldots x_{i-1} x_{i+1} \ldots x_{m} \text { for } i=1, \ldots, m .
$$

Thus $X_{\infty}$ is a quotient of the disjoint union $\coprod_{m \geqslant 0} X^{m}$ and this defines the topology. The space $X_{\infty}$ is based at the empty word which we also denote by $*$. A point of $X_{\infty}$ has a unique representation as a reduced word, that is, one with $x_{i} \neq *$ for all $i$.

\section{The James map}

Let $X$ be a based topological space and suppose that there is a map $\rho_{X}: X \rightarrow I$ with the property that $\rho_{X}^{-1}(0)=\{*\}$. Let $\Omega(X)$ denote the loop space of $X$ and $S(X)=X \wedge S^{1}$ the suspension of $X$. (Notice that we take the suspension coordinate second.) We shall identify $S^{1}$ with $I / \partial I$. Thus a point of $S^{1}-\{*\}$ is uniquely represented as a real number $t$ with $0<t<1$, and a point of $S(X)-\{*\}=\left(X \wedge S^{1}\right)-\{*\}$ is uniquely represented as a pair $(x, t)$ where $x \in X-\{*\}$ and $0<t<1$. It is convenient to regard the suspension coordinate as vertical.

Suppose given a non-empty reduced word $x_{1} \ldots x_{m} \in X_{\infty}$ where each $x_{i} \neq *$. Let $\alpha_{i}=\rho_{X}\left(x_{i}\right)$, and let $t_{i}$ be given by $t_{0}=0$ and

$$
t_{i}=\frac{\alpha_{1}+\ldots+\alpha_{i}}{\alpha_{1}+\ldots+\alpha_{m}}
$$

for $0<i \leqslant m$. Then $0=t_{0}<t_{1}<\ldots<t_{m}=1$.

The James map $k_{X}: X_{\infty} \rightarrow \Omega S(X)$ is given by $k_{X}(*)=*$ and for non-empty words

$$
k_{X}\left(x_{1} \ldots x_{m}\right)(t)=\left(x_{i}, \frac{t-t_{i-1}}{t_{i}-t_{i-1}}\right) \text {, }
$$

for $t_{i-1} \leqslant t \leqslant t_{i}$.

In words, what $k_{X}$ does is to map the word $x_{1} \ldots x_{m}$ to a loop in $S(X)$ which comprises $m$ vertical loops passing through $x_{1}, \ldots, x_{m}$ respectively, with the time parameters adjusted by using $\rho$ to make the time spent on a subloop go to zero as the corresponding point $x_{i}$ moves to the base-point of $X$.

James proves that $k_{X}$ is a homotopy equivalence if $X$ is a countable CW complex with just one vertex. However the result extends to a considerably more 
general class of spaces, $[\mathbf{2 1}]$. We shall be particularly interested in the case when $X$ is the $n$-sphere and we shall abbreviate $k_{S^{n}}$ to $k_{n}$.

\section{The classical James-Hopf invariants}

We make the following further identifications:

$$
S^{n+1}=S\left(S^{n}\right)=S^{n} \wedge S^{1}=S^{1} \wedge \ldots \wedge S^{1}=I / \partial I \wedge \ldots \wedge I / \partial I=I^{n+1} / \partial I^{n+1} .
$$

This means that a point of $S^{n}-\{*\}$ is uniquely an $n$-tuple $x=\left(x_{1}, x_{2}, \ldots, x_{n}\right)$ where $0<x_{i}<1$.

We choose the map $\rho_{S^{n}}$ rather carefully.

Let $\rho: I \rightarrow I$ be a smooth bump function with the property that $\rho^{-1}(0)=\{0,1\}$ and all derivatives vanish at 0 and 1 . Let $\rho_{S^{1}}: S^{1} \rightarrow I$ be the induced map, and define $\rho_{S^{n}}: S^{n} \rightarrow I$ by $\rho_{S^{n}}\left(x_{1}, x_{2}, \ldots, x_{n}\right)=\rho\left(x_{1}\right) \rho\left(x_{2}\right) \ldots \rho\left(x_{n}\right)$.

Now let $g_{n}: S_{\infty}^{1} \rightarrow S_{\infty}^{n}$ be given by

$$
g_{n}\left(x_{1} \ldots x_{m}\right)=\prod_{\lambda}\left(x_{\lambda_{1}}, \ldots, x_{\lambda_{n}}\right),
$$

where the product is over all strictly monotone $\lambda:\{1, \ldots, n\} \rightarrow\{1, \ldots, m\}$ and is taken in lexicographical order.

The $n$th James-Hopf invariant $J_{n}:\left[S(X), S^{2}\right] \rightarrow\left[S(X), S^{n+1}\right]$ is induced by the composition

$$
\Omega S^{2} \stackrel{\tau}{\longrightarrow} S_{\infty}^{1} \stackrel{g_{n}}{\longrightarrow} S_{\infty}^{n} \stackrel{k_{n}}{\longrightarrow} \Omega S^{n+1}
$$

where $\tau$ is a homotopy inverse to $k_{1}$.

\section{The trivial $\square$-set $T$}

We now turn to the connection between embedded James bundles and the James-Hopf invariants. We start by identifying the homotopy type of the trivial $\square$-set $T$ (with exactly one cell in each dimension).

3.3. Proposition. The realisation of the $\square$-set $T$ can be identified with $S_{\infty}^{1}$ and hence has the homotopy type of $\Omega\left(S^{2}\right)$.

Proof. Denote the unique $m$-cube of $T$ by $c_{m}$. Let $x \in|T|$. Then $x \in \operatorname{int}\left(c_{m}\right)$ for some $m$ and hence $x$ has a unique expression as $\left(x_{1}, \ldots, x_{m}\right)$ where each $0<x_{i}<1$. Moreover the glue given by the face maps has the effect of omitting any $x_{i}$ which become 0 or 1 . It follows that the map $x=\left(x_{1}, \ldots, x_{m}\right) \mapsto x_{1} \ldots x_{m}$ is a homeomorphism $|T| \rightarrow S_{\infty}^{1}$.

REMARK. Antolini [1] has generalised this result and shown that the singular $\square$-set of any topological space $X$ has the weak homotopy type of $X \times \Omega\left(S^{2}\right)$; see also $[2]$.

\section{The James bundles of $T$}

Now consider the $n$th James bundle $\zeta^{n}(T)$. In the last section we showed that it embeds as a framed mock bundle in $|T| \times \mathbb{R}$. If we apply the Thom-Pontrjagin construction to this framed embedding, we obtain a map $|T| \times \mathbb{R} \rightarrow S^{n+1}$ and 
hence a map $q_{n}: S(|T|) \rightarrow S^{n+1}$. Using the last proposition we have a map

$$
q_{n}: S\left(S_{\infty}^{1}\right) \longrightarrow S^{n+1} .
$$

The following proposition connects the James bundles of $T$ with the James-Hopf invariants.

3.4. Proposition. The framed embedding of $E_{\zeta^{n}(T)}$ in $|T| \times \mathbb{R}$ can be chosen so that $q_{n}$ is the adjoint of

$$
S_{\infty}^{1} \stackrel{g_{n}}{\longrightarrow} S_{\infty}^{n} \stackrel{k_{n}}{\longrightarrow} \Omega\left(S^{n+1}\right) .
$$

Proof. Let $h_{n}: I^{n+k} \times I \rightarrow S^{n+1}$ be the map determined by the adjoint of $k_{n} g_{n}$ restricted to the $(n+k)$-cell of $T$. Then $h_{n}$ is transverse to $a=\left(\frac{1}{2}, \ldots, \frac{1}{2}\right)$ and we shall see that the framed submanifold $h^{-1}(a)$ can be identified with the block of $E_{\zeta^{n}(T)}$ over $I^{n+k}$. Moreover the restriction of $h_{n}$ to $h_{n}^{-1}\left(\operatorname{int}\left(I^{n+1}\right)\right)$ provides a trivialisation of an open tubular neighbourhood of $E_{\zeta^{n}(T)}$ in $I^{n+k} \times I \subset I^{n+k} \times \mathbb{R}$. The closure of this neighbourhood is the whole of $I^{n+k} \times I$. Figure 5 illustrates the case $n=k=1$. Only the vertical part of the framing is shown for clarity. In general the $k$-cubes $M_{c_{\lambda}}$ are placed in lexicographical order above their images $s_{\lambda}\left(I^{k}\right)$ in $I^{n+k}$.

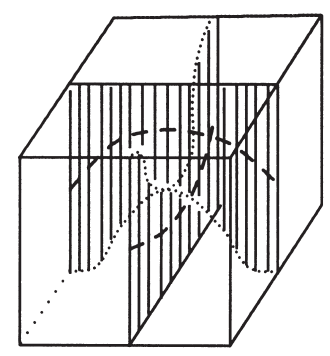

FiguRE 5.

We now give a precise formula for the embedding of this block and then the statements made above can all be checked. Let $\lambda^{1}, \ldots, \lambda^{N}$ be the list of $\lambda \mathrm{s}$ in the definition of $g_{n}$ (equation (3.2)) with $m=n+k$, taken in order. Then from (3.1) and (3.2) we have

$$
h_{n}\left(x_{1}, \ldots, x_{n+k}, t\right)=\left(x_{\lambda_{1}^{i}}, \ldots, x_{\lambda_{n}^{i}}, \frac{t-t_{i-1}}{t_{i}-t_{i-1}}\right)
$$

where

$$
t_{i}=\frac{\alpha_{1}+\ldots+\alpha_{i}}{\alpha_{1}+\ldots+\alpha_{N}} \quad \text { for } 0 \leqslant i \leqslant N
$$

and $\alpha_{i}=\rho_{n}\left(x_{\lambda_{1}^{i}}, \ldots, x_{\lambda_{n}^{i}}\right)$. Each $\lambda^{i}=\left(\lambda_{1}^{i}, \ldots, \lambda_{n}^{i}\right)$ corresponds to a $k$-cube $M_{c_{\lambda^{i}}}$ of the $n$th associated James complex, namely the cube $\left(c_{n+k}, \lambda^{i}\right) \in J^{n}(T)_{k}$, in the block of $E_{\zeta^{n}(T)}$ over $c_{n+k}$. Recall that the $\lambda_{j}^{i}$ index the directions in $I^{n+k}$ normal to the $k$-cube $\left(c_{n+k}, \lambda^{i}\right)$. 
We will define an embedding

$$
e_{\lambda^{i}}: I^{k} \times \operatorname{int}\left(I^{n}\right) \times \operatorname{int}(I) \longrightarrow I^{n+k} \times I \subset I^{n+k} \times \mathbb{R} .
$$

Let $s h_{\lambda^{i}}: I^{n+k} \rightarrow I^{n+k}$ be the isometry given by permuting coordinates as follows: $s h_{\lambda^{i}}$ sends the $(k+s)$ th coordinate to the $\lambda_{s}^{i}$ th coordinate, for $1 \leqslant s \leqslant n$, and preserves order on the remaining $k$ coordinates. Write $s h_{\lambda^{i}}(y)=x$; then

$$
e_{\lambda^{i}}(y, u)=\left(x, u t_{i}+(1-u) t_{i-1}\right) .
$$

Note that the images of the $e_{\lambda^{i}}$ are disjoint.

Now define the embedding of the $k$-cube $\left(c_{n+k}, \lambda^{i}\right) \in J^{n}(T)_{k}$ by identifying $I^{k}$ with $I^{k} \times\left(\frac{1}{2}, \ldots, \frac{1}{2}\right) \times \frac{1}{2} \subset I^{k} \times \operatorname{int}\left(I^{n}\right) \times \operatorname{int}(I)$ and composing $e_{\lambda^{i}}$ with $\chi_{c_{n+k}} \times \operatorname{id}_{\mathbb{R}}$. If we do this for each of the $\lambda^{i}$, we obtain a framed embedding of the block of $\zeta^{n}(T)$ over $c_{n+k}$ and the required properties follow from comparing the formulæ above with (3.1) and (3.2).

REMARK. The embeddings $e_{\lambda^{i}}$ above extend to maps (not embeddings) of $I^{k} \times I^{n} \times I \rightarrow I^{n+k} \times \mathbb{R}$ whose images fill the whole of $I^{n+k} \times I$. However the Thom-Pontrjagin construction works for such framings, since the boundary of the tubular neighbourhood is mapped to the base-point. If a closed trivial tubular neighbourhood is required, then we can restrict to a small $(k+1)$-cube at the centre of $I^{k} \times I$.

We now turn to general $\square$-sets. The following proposition follows at once from definitions.

3.5. Proposition. James bundles are natural, that is, given a $\square$-map $f: C \rightarrow D$ then $\zeta^{n}(C)$ is the pull-back $f^{*}\left(\zeta^{n}(D)\right)$.

\section{The James-Hopf invariants of a $\square$-set}

If $C$ is any $\square$-set, there is a canonical constant $\square$-map $t_{C}: C \rightarrow T$; so Proposition 3.5 implies that $\zeta^{n}(C)=t_{C}^{*} \zeta^{n}(T)$. Further, the framed embedding of $\zeta^{n}(T)$ in $|T| \times \mathbb{R}$ pulls back to a framed embedding of $\zeta^{n}(C)$ in $|C| \times \mathbb{R}$ which (by the Thom-Pontrjagin construction) determines a map $S(C) \rightarrow S^{n+1}$.

We call the homotopy class of this map $j_{n}(C) \in\left[S(C), S^{n+1}\right]$ the $n$th James-Hopf invariant of the $\square$-set $C$.

3.6. TheOREM. (1) The James-Hopf invariants of a $\square$-set are natural, that is, given a $\square$-map $f: C \rightarrow D$ then $j_{n}(C)=S(f)^{*} j_{n}(D)$.

(2) If we identify $T$ with $S_{\infty}^{1}$ by Proposition 3.3 and with $\Omega\left(S^{2}\right)$ via the homotopy equivalence $k_{1}$ then the classical James-Hopf invariant $J_{n}$ (defined earlier) is induced by composition with $j_{n}(T)$.

(3) Let $q_{C}: S(C) \rightarrow S^{2}$ denote the adjoint of

$$
C \stackrel{t_{C}}{\longrightarrow} T=S_{\infty}^{1} \stackrel{k_{1}}{\longrightarrow} \Omega\left(S^{2}\right) .
$$

Then $j_{n}(C)=J_{n}\left(q_{C}\right)$. 
Proof. Naturality follows at once from definitions. Moreover, note that (2) is a restatement of Proposition 3.4, after observing that $q_{n} \equiv j_{1}(T)$ (the notation $q_{n}$ was used in Proposition 3.4). Finally,

$$
J_{n}\left(q_{C}\right)=\operatorname{adj}\left(\left(k_{n} g_{n}\right) t_{C}\right)=S\left(t_{C}\right)^{*} \operatorname{adj}\left(k_{n} g_{n}\right)=S\left(t_{C}\right)^{*} j_{n}(T)=j_{n}(C) .
$$

3.7. REMARK. In [12] we shall relate James bundles to multiple points of immersions: if $f: M \rightarrow C$ is a transverse map, then the Poincaré duals of the pull-backs of the James bundles comprise a self-transverse immersion and its multiple manifolds. Using this result, we see that the connection we have given of James bundles with classical James-Hopf invariants overlaps with the results of Koschorke and Sanderson [17] which also relate generalised James-Hopf invariants to multiple points of immersions. The results overlap when considering $\Omega S\left(S^{1}\right)$. Here $\Omega S\left(S^{1}\right)$ is a special case of $|C|$. In $[\mathbf{1 7}], \Omega S\left(S^{1}\right)$ is a special case of $\Omega^{n} S^{n}(T(\xi))$, with $n \geqslant 1$, where $T(\xi)$ is a Thom space.

\section{James classes and characteristic classes}

In this section we examine the algebra generated by the James classes and recover (stably) the results of Baues [4]. We also consider the Hurewicz images in ordinary cohomology, and hence define natural characteristic classes for a $\square$-set whose mod 2 reductions are pulled back from both the Stiefel-Whitney and the $\mathrm{Wu}$ classes of $B O$.

The James-Hopf invariants $j_{n}(C)$ of a $\square$-set $C$ are classes in unstable cohomotopy, which stabilise to classes $\gamma_{n}(C) \in \mathbb{S}^{n}(C)$ in stable cohomotopy. There is a cup product defined for the unstable classes. Baues [4, p. 79] gives a formula for these cup products for the case $C=T$ (that is, for $S_{\infty}^{1}$ ). See Dreckmann [7, p. 42] for a correction. Here we give a simple geometric proof of the formula after one suspension.

The (unstable) cup product

$$
\left[S X, S^{m+1}\right] \times\left[S X, S^{n+1}\right] \longrightarrow\left[S X, S^{n+m+1}\right]
$$

may be described in terms of mock bundles as follows. Let $\xi^{m}$ and $\eta^{n}$ be framed mock bundles embedded in $(X-\{*\}) \times \mathbb{R}$. Assume the projections $p_{\xi}$ and $p_{\eta}$ are transverse. Define $E_{\xi \cup \eta}=p_{\xi}^{*} E_{\eta}$. Since $E_{\eta}$ is $(n+1)$-framed in $X \times \mathbb{R}, E_{\xi \cup \eta}$ is $(n+1)$-framed in $E_{\xi} \times \mathbb{R}$. But $E_{\xi}$ is $(m+1)$-framed in $X \times \mathbb{R}$, so using the last of these framing directions to embed $E_{\xi} \times \mathbb{R}$ in $X \times \mathbb{R}$ we have an embedding of $E_{\xi \cup \eta}$ which is $(n+m+1)$-framed in $X \times \mathbb{R}$. If the roles of $\xi$ and $\eta$ are reversed we get a homeomorphic result with a possible framing change.

However, in the case of the James bundles, the last framing direction is parallel to $\mathbb{R}$ in $X \times \mathbb{R}$ and we can see that the two cup products agree up to permutation of components of blocks and a sign change. They also agree (after one suspension and up to sign) with the external cup product (take the Cartesian product, which is naturally $(n+m+2)$-framed in $X^{2} \times \mathbb{R}^{2}$ and restrict to the diagonal). This is seen by rotating the last framing vector through a right angle into the last $\mathbb{R}$ coordinate.

Now let $H$ be an $n$ element subset of $\{1,2, \ldots, m+n\}$. Let $s(H)$ be the sign of the shuffle permutation of $\{1,2, \ldots, m+n\}$ which moves $H$ to the front and 
preserves order in both $H$ and its complement. Let $\phi_{m, n}=\sum_{H} s(H)$ where the sum runs over all $n$-element subsets $H$ of $\{1,2, \ldots, m+n\}$.

\subsection{Proposition. For any $\square$-set $C$,}

$$
S j_{m}(C) \cup S j_{n}(C)=\phi_{m, n} S j_{m+n}(C) .
$$

Proof. We prove the result in the case $C=T$, the trivial complex. The formula then pulls back over the constant map $t_{C}: C \rightarrow T$ since the cup product is natural for cubical maps. To compute the cup product we must make the mock bundle projections $p_{m}:\left|J^{m}(T)\right| \rightarrow|T|$ and $p_{n}:\left|J^{n}(T)\right| \rightarrow|T|$ transverse. Let $b_{1}=c_{\left(i_{1}, \ldots, i_{m}\right)}$ and $b_{2}=c_{\left(l_{1}, \ldots, l_{n}\right)}$ be elements of $J^{m}(T)$ and $J^{n}(T)$ respectively where $c \in T_{k}$. In order to compute the transverse intersection of $p_{m}\left|b_{1}\right|$ with $p_{n}\left|b_{2}\right|$ in $|c|$ consider the corresponding sections $s_{\left(i_{1}, \ldots, i_{m}\right)}: I^{k-m} \rightarrow I^{k}$ and $s_{\left(l_{1}, \ldots, l_{n}\right)}: I^{k-n} \rightarrow I^{k}$. The contribution to $j_{m}(T) \cup j_{n}(T)$ over $|c|$ is given as follows. There are two possibilities. If $\left\{i_{1}, \ldots, i_{m}\right\} \cap\left\{l_{1}, \ldots, l_{n}\right\}=\emptyset$ then the sections are transverse and they meet in the image of $s_{\left(p_{1}, \ldots, p_{n+m}\right)}$ where

$$
\left\{p_{1}, \ldots, p_{n+m}\right\}=\left\{i_{1}, \ldots, i_{m}\right\} \cup\left\{l_{1}, \ldots, l_{n}\right\} .
$$

Since, strictly speaking, $p$ is a function $\{1, \ldots, n+m\} \rightarrow \mathbb{N}$ we can define $H=p^{-1}\left\{i_{1}, \ldots, i_{m}\right\}$. We have determined an element $c_{\left(p_{1}, \ldots, p_{n+m}\right)} \in J^{n+m}(T)$ whose realisation is a $k-(n+m)$ cube in the total space of the mock bundle $\zeta^{n+m}(T)$. But the cup product framing differs from the natural framing by the sign of the shuffle $s(H)$. This covers the transverse case.

If say $\left\{i_{1}, \ldots, i_{m}\right\} \cap\left\{l_{1}, \ldots, l_{n}\right\}=\left\{q_{1}, \ldots, q_{s}\right\}$ with $q_{1}<\ldots<q_{s}$ then we isotope $p_{n}$ on $\left|c_{\left(j_{1}, \ldots, j_{n}\right)}\right|$ by increasing the $q_{1}$ th coordinate in $I^{k}$ from $\frac{1}{2}$ to say $\frac{3}{4}$, then the intersection is empty and there is no contribution to $j^{m} j^{n}$ in this case.

At this point we have proved the result provided we can cancel off contributions of opposite sign and reorder the pieces. For this we need to use the one extra dimension where there are obvious cobordisms which achieve this result.

4.2. Lemma. For $m, n \in \mathbb{N}$,

$$
\phi_{m, n}= \begin{cases}\left(\begin{array}{c}
{[(m+n) / 2]} \\
{[n / 2]}
\end{array}\right) & \text { if } n m \text { is even } \\
0 & \text { otherwise. }\end{cases}
$$

In particular for all $m$ and $n$,

$$
\phi_{2 m, 2 n}=\left(\begin{array}{c}
m+n \\
m
\end{array}\right), \quad \phi_{m, n}=\left(\begin{array}{c}
m+n \\
m
\end{array}\right) \bmod 2, \quad \phi_{1,1}=0, \quad \phi_{1,2 n}=1 .
$$

Proof. Clearly $\phi_{0, n}=\phi_{m, 0}=1$. Set $\phi_{m, n}=0$ if either $m$ or $n$ is negative. For $\phi_{m, n}$ the sum can be split up, according as the least element of $H$ is or is not 1 , giving the recurrence relation

$$
\phi_{m, n}=\phi_{m, n-1}+(-1)^{n} \phi_{m-1, n} .
$$

The result now follows by induction. 
The computation of the algebra generated by the stabilisation of the James-Hopf invariants easily follows. We shall carry out this computation after introducing the characteristic classes of a $\square$-set, which are the corresponding cohomology classes.

The characteristic classes of a $\square$-set

Let $C$ be any $\square$-set. The $n$th characteristic class of $C, V_{n}(C) \in H^{n}(C)$, is the class of the unit cocycle: value 1 on each $n$-cube.

Let $\mathbb{S}$ denote the sphere spectrum and let $\gamma_{n}(C) \in \mathbb{S}^{n}(C)$ denote the stabilisation of $j_{n}(C)$. The element $\gamma_{n}(C)$ is represented by $E_{\zeta^{n}}$ framed in $|C| \times \mathbb{R}^{\infty}$. Let $h: \mathbb{S}^{*} \rightarrow H^{*}$ be the Hurewicz transformation.

\subsection{Proposition. For any $\square$-set $C$,}

$$
h\left(\gamma_{n}(C)\right)=V_{n}(C) .
$$

Proof. At the mock bundle level $h$ can be described simply by counting, with sign, the components of the 0-dimensional blocks. The result follows since the 0 -dimensional blocks of $\gamma_{n}$ consist of a single point over each $n$-cube with standard framing.

Denote the exterior algebra, over $\mathbb{Z}$, on one generator $x$ by $E(x)$; then $x^{2}=0$ and elements of $E(x)$ can be written as $p+q . x$, where $p, q \in \mathbb{Z}$. Denote the divided polynomial algebra on one generator $y$ by $D(y)$; in other words, there are elements $y_{n}$, for $n \geqslant 0$, with $y_{0}=1$ and $y_{1}=y$ such that

$$
y_{n} \cdot y_{m}=\left(\begin{array}{c}
n+m \\
m
\end{array}\right) y_{n+m} \text {. }
$$

An element of $D(y)$ is then just a linear combination of the $y_{n}$.

4.4. Corollary. The elements $\gamma_{n}(T)$, with $n \geqslant 0$, generate a subalgebra of $\mathbb{S}^{*}(|T|)$ of form $E\left(\gamma_{1}(T)\right) \otimes D\left(\gamma_{2}(T)\right)$. In particular any element of the subalgebra can be uniquely written as a linear combination of the elements $\left\{1, \gamma_{1} \gamma_{2 n}, \gamma_{2 n}\right\}_{n \geqslant 0}$.

Proof. The result follows from Proposition 4.1 and Lemma 4.2 and the fact noted above that the Hurewicz map $h: \mathbb{S}^{*}(|T|) \rightarrow H^{*}(|T|)$ is non-trivial on the $\gamma_{n}$ for $n \geqslant 0$. In fact it follows that $h$ restricted to the subalgebra is an isomorphism.

The $\mathbb{Z}_{2}$-characteristic classes

Let $v_{n}$ denote the mod 2 reduction of our characteristic class $V_{n}$. We can identify $v_{n}(T)$ as the Stiefel-Whitney class of a vector bundle. Background for what follows can be found in $[\mathbf{2 3}]$ and $[\mathbf{1 9}]$. Let $B O$ be the classifying space for the infinite orthogonal group, and let $B^{2} O$ be its delooping; so $\Omega B^{2} O \simeq B O$. Let $\omega: S^{2} \rightarrow B^{2} O$ be a generator of $\pi_{2} B^{2} O \cong \mathbb{Z}_{2}$. By taking $\Omega \omega$ we get a map $s:|T| \rightarrow B O$ with the property that $s$ restricted to the 1 -skeleton classifies the stable unoriented bundle. 
4.5. Proposition. We have $w_{n}\left(s^{*} \gamma\right)=w_{n}\left(s^{*} \gamma^{-1}\right)=v_{n}(T)$, for $n \geqslant 0$, where $\gamma$ is the universal (virtual) bundle.

Proof. Consider the $n$-fold product $S_{n}=S^{1} \times \ldots \times S^{1}$. Let $m: S_{n} \rightarrow|T|$ be given by multiplication. Then $m^{*}: H^{n}(|T|) \rightarrow H^{n}\left(S_{n}\right)$ is an isomorphism. Since $s$ is an $H$-map, $w\left(m^{*} s^{*} \gamma\right)=\prod_{i=1}^{n}\left(1+a_{i}\right)$ where each $a_{i}$ is the generator of $H^{1}\left(S^{1}, \mathbb{Z}_{2}\right)$, and so $w_{n}\left(s^{*} \gamma\right) \neq 0$. Since $m^{*} s^{*} \gamma$ is a product of (stable) line bundles over $S^{1} \mathrm{~s}, m^{*} s^{*} \gamma=m^{*} s^{*} \gamma^{-1}$.

Let $\phi_{C}:|C| \rightarrow B O$ be the composition $s \circ t$ where $t:|C| \rightarrow|T|$ and let $\gamma_{C}=\phi_{C}^{*} \gamma$. We call $\gamma_{C}$ the characteristic bundle over $C$.

\subsection{Corollary. For any $\square$-set $C, w_{n}\left(\gamma_{C}\right)=v_{n}(C)$ for $n \geqslant 0$.}

REMARK. Recall that the (total) Stiefel-Whitney class, $w(\xi)$, of a vector bundle $\xi$ is defined by $w(\xi) \cup U=S q U$ where $U$ is the Thom class. Similarly the $\mathrm{Wu}$ class, $\mathrm{wu}(\xi)$, is given by $\mathrm{wu}(\xi) \cup U=\chi S q U$. From this and the Cartan formula we get the well-known relation $S q \mathrm{wu}(\xi)=w\left(\xi^{-1}\right)$. On $S_{n}$ we have $S q=\mathrm{Id}$ and $m^{*} s^{*} \gamma=m^{*} s^{*} \gamma^{-1}$ so in both Proposition 4.5 and Corollary 4.6, Stiefel-Whitney and $\mathrm{Wu}$ classes coincide.

We shall continue to study the characteristic bundle and the associated map $\phi_{C}:|C| \rightarrow B O$ in the next section, where we use it to define the concept of a $C$-oriented manifold and to define an associated generalised cohomology theory.

\section{Homology theories from $\square$-sets}

In this section we describe how a $\square$-set may be used to construct homology and cohomology theories. More precisely we shall define a spectrum $M C$ for any $\square$-set $C$ which then defines a generalised homology theory $M C_{*}$. This theory has a good description as a bordism theory in terms of manifolds and labelled diagrams. The description is particularly nice when $C$ is the classifying space of a rack. These theories have natural operations, which as we shall see are analogous to $\mathcal{X} S q$ operations in ordinary cohomology.

In the case $C=T$, the trivial $\square$-set, the spectrum $M T$ is a ring spectrum and is one of a family studied by Mahowald [19].

\section{The spectrum $M C$}

We recall from $\S 4$ that there is a map $s:|T| \rightarrow B O$ (where $T$ is the trivial $\square$-set with just one cell in each dimension and $B O$ is the classifying space for stable vector bundles) and this defines a map $\phi_{C}=s \circ t_{C}:|C| \rightarrow B O$ for any $\square$-set $C$, where $t_{c}: C \rightarrow T$ is the canonical $\square$-map. The map $\phi_{C}$ has the property that it pulls both the universal Stiefel-Whitney and $\mathrm{Wu}$ classes back to the mod 2 reductions of the characteristic classes of $C$; see Corollary 4.6.

Let $\gamma$ be the universal (virtual) bundle over $B O$ and $\gamma_{C}$ (the characteristic bundle) its pull-back over $C$ by $\phi_{C}$. The spectrum $M C$ is defined to be the associated Thom spectrum to $\gamma_{C}$. It defines a generalised homology theory, which we call $C$-bordism, and denote $M C_{*}$ and a corresponding theory of $C$-cobordism denoted $M C^{*}$. 


\section{Connection with standard spectra}

We next see how the new spectrum fits with the sphere spectrum (which classifies stable cohomotopy) and the Eilenberg-MacLane spectrum which classifies ordinary $(\bmod 2)$ cohomology.

The universal Thom class determines a map of spectra $M O \rightarrow H \mathbb{Z}_{2}$, where $H \mathbb{Z}_{2}$ is the mod 2 Eilenberg-MacLane spectrum. Hence we get a natural map $\theta_{C}: M C \rightarrow H \mathbb{Z}_{2}$. Let $\mathbb{S}$ denote the sphere spectrum, and let $\mathbb{S Z}_{2}$ denote the sphere spectrum with mod 2 coefficients.

\subsection{Proposition. For any non-empty $\square$-set $C$ :}

(1) the Hurewicz map factors as $\mathbb{S} \rightarrow M C \rightarrow H \mathbb{Z}_{2}$;

(2) if there is an element $x \in C_{1}$ with $\partial_{1}^{0} x=\partial_{1}^{1} x$ then the mod 2 Hurewicz map factors as $\mathbb{S Z}_{2} \rightarrow M C \rightarrow H \mathbb{Z}_{2}$.

Note. The condition in part (2) is always satisfied for non-empty rack spaces.

Proof. The sphere spectrum $\mathbb{S}$ may be regarded as the Thom spectrum pulled back from $B O$ by the inclusion of a point. The first result follows from naturality of $\theta$ by choosing a vertex in $|C|$.

For the second part let $D$ be the $\square$-set which is the circle with the obvious $\square$-set structure, that is, $D$ has a single vertex, a single edge and no higher cells. From the property that $\phi_{D}$ pulls back the Stiefel-Whitney class to the mod 2 characteristic class of $D$, it can be seen that $\phi_{D}:|D| \rightarrow B O$ classifies the (stable) Möbius band over the circle $|D|$. The Thom complex of the Möbius band is the projective plane and $M D$ is its suspension spectrum, that is, $\mathbb{S Z}_{2}$. The transformation $\theta_{D}: M D \rightarrow H \mathbb{Z}_{2}$ can now be identified with the inclusion of the (stable) 1-skeleton, that is, the mod 2 Hurewicz map. The result now follows from naturality of $\theta$ by considering the inclusion of $D$ in $C$ given by mapping the edge of $D$ to $x$.

\section{Twisted bundles}

The generalised homology theory defined by $M C$ is the bordism theory defined by a certain class of manifolds called $C$-manifolds. We shall describe this class.

We need to define what it means for a manifold to have its normal bundle twisted by a self-transverse immersed submanifold of codimension 1. For simplicity we describe the case of an embedding first. So let $Q \subset M$ be a codimension 1 submanifold and assume for simplicity that $Q$ is equipped with a bicollar neighbourhood $N(Q)$. Denote by $\rho / I$ the Möbius bundle which is the 1-dimensional vector bundle constructed by gluing the trivial bundles over $\left[0, \frac{1}{2}\right]$ and $\left[\frac{1}{2}, 1\right]$ by multiplication by -1 in the fibre over $\frac{1}{2}$. Thus although $\rho / I$ is the trivial bundle, it is equipped with opposite trivialisations on the two halves of $I$. Denote by $\hat{\rho} / I$ the stable version. We call $\hat{\rho} / I$ the (stable) Möbius twist.

Now construct the stable bundle $\hat{\rho} / M$ by taking the trivial bundle over $Q^{c}=\overline{M-N(Q)}$ and gluing in $\widehat{\rho} / I$ on each collar line. Alternatively, we can take the trivial line bundle on $M-Q$ and glue at $Q$ by multiplication by -1 and then stabilise. We say that the stable normal bundle $\nu / M$ of $M$ is twisted by $Q$ if it is identified with $\widehat{\rho} / M$. 
The concept of twisting by a self-transverse immersion is similar except that at $k$-tuple points there are $k$ mutually perpendicular Möbius twists taking place corresponding to the directions perpendicular to the $k$ sheets.

For the detailed definition we shall construct a stable Möbius twist bundle over $I^{n}$ for each $n$. To do this we need a function $v: J^{1}\left(I^{n}\right) \rightarrow S^{n-1}$ with an orthogonality property. Denote $v \mid I_{(i)}^{n}$ by $v_{i}$ which we think of as a variable unit vector in $\mathbb{R}^{n}$. We require that, for each point $p \in I^{n}$ covered by points $p_{i_{1}}, \ldots, p_{i_{t}}$ in $I_{\left(i_{1}\right)}^{n}, \ldots, I_{\left(i_{t}\right)}^{n}$, the corresponding vectors $v_{i_{1}}, \ldots, v_{i_{t}}$ should form an orthonormal $t$-frame. We also require that $v$ commutes with face maps, that is, the following diagram commutes:

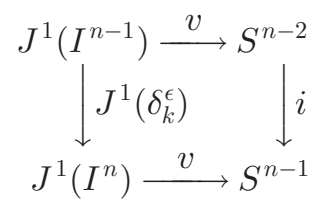

where $i: S^{n-2} \rightarrow S^{n-1}$ is the usual inclusion.

We define $v$ by induction on $n$ and suppose that $v: J^{1}\left(I^{n-1}\right) \rightarrow S^{n-2}$ is already defined. Then the commuting diagram defines $v$ on $\partial I^{n}$. We extend to the interior of $I^{n}$ by using the cubical subdivision of $I^{n}$ determined by the image of $J^{1}\left(I^{n}\right)$ in $I^{n}$, which cuts $I^{n}$ into $2^{n}$ subcubes. Note that $n-i$ sheets of the image of $J^{1}$ meet at each $i$-cube in int $I^{n}$ in this subdivision (see the diagram near the end of $\S 1$ ). We define $v$ by induction over skeleta. At the unique 0 -cell in $\operatorname{int} I^{n}$ we choose $v_{1}, \ldots, v_{n}$ to be any orthonormal $n$-frame. In general we have an orthonormal $(n-i)$-frame defined over the boundary of a typical $i$-cell and we extend over the $i$-cell using the fact that $\pi_{i-1}\left(V_{n, n-i}\right)=0$.

This completes the definition of $v$. Notice that the choices made in the definition are homotopic if $S^{n-1}$ is replaced by $S^{n}$ and hence the stable twisted bundles constructed below do not depend on these choices.

We now construct the $n$-dimensional vector bundle $\rho / I^{n}$ by taking the trivial bundle off the image of $J^{1}$ and gluing by mapping $v_{i} \mapsto-v_{i}$ as the $i$ th sheet (the image of $\left.I_{(i)}^{n}\right)$ is crossed and leaving the orthogonal complement of $v_{i}$ fixed. Near a point $p \in I^{n}$ covered by points $p_{i_{1}}, \ldots, p_{i_{t}}$ in $I_{\left(i_{1}\right)}^{n}, \ldots, I_{\left(i_{t}\right)}^{n}$ the various trivial bundles are being glued by reversing the signs of the appropriate subset of $v_{i_{1}}, \ldots, v_{i_{t}}$. By orthogonality, these $t$ simultaneous gluings are all independent. We denote the stable version of $\rho / I^{n}$ by $\widehat{\rho}^{n}$. This is the $n$-fold Möbius twist over $I^{n}$.

By construction, Möbius twists have the property that for each face map $\lambda: I^{t} \rightarrow I^{p}, \lambda^{*}\left(\widehat{\rho}^{p}\right)=\widehat{\rho}^{t}$. This implies that if $X$ is a $\square$-space then we can construct a bundle $\hat{\rho} /|X|$ by gluing together the bundles $X_{n} \times \widehat{\rho}^{n}$ mimicking the definition of $|X|$. Note that $\hat{\rho} /|X|$ is trivialised over $X_{0}$ and at $X_{n}$ there is an $n$-fold Möbius twist taking place.

\section{Neighbourhood systems and transversality}

Now suppose that $Q$ is the image of a self-transverse [18] immersed submanifold of $M^{m}$ of codimension 1 . We can construct a neighbourhood system $N(Q)$ as follows. We choose a cube neighbourhood of each $m$-tuple point, which (using self-transversality) can be assumed to meet nearby sheets in the $m$ central $(m-1)$-subcubes (that is, the images of $\left.J^{1}\right)$. Then, working in the complement of the interiors of these cubes we choose $I^{m-1}$ bundle neighbourhoods of the 
1-dimensional $(m-1)$-tuple points which meet the cubes near the $m$-tuple points in faces. We continue to choose inductively $I^{q}$ bundle neighbourhoods of the $q$-tuple points meeting nearby sheets in central subcubes and higher multiple points in faces. We finish by choosing (trivial) $I^{0}$ bundle structures at the 0 -tuple points (that is, the points of $M-Q$ ). Thus the final neighbourhood system decomposes $M$ into $I^{n}$ bundles for varying $n$, glued together along the analogue of faces.

We need the following theorem.

5.2. Transversality Theorem. Let $C$ be a $\square$-set and $f: M^{m} \rightarrow|C|$ a map. Then $f$ is homotopic to a map $f^{\prime}$ (called transverse) which has the following property. There are a self-transverse immersed submanifold $Q \subset M$ of codimension 1 and a neighbourhood system $N(Q)$ which is in fact the realisation of a $\square$-space such that $f^{\prime}: M \rightarrow|C|$ is the realisation of a $\square$-map.

Proof. The proof is similar to the proof of transversality for CW complexes given in [6, Chapter 7]. We construct $f^{\prime}$ by a sequence of changes using a downwards induction on the dimension of skeleta of $C$ containing the image of $f$. By the usual abuse of notation we will continue to call the amended maps $f$. By cellular approximation we can assume that the image of $f$ lies in the $m$-skeleton of $C$. Use smooth transversality to make $f$ transverse to the centres of the $m$-cubes of $C$ and then by expanding a small neighbourhood of each centre onto the whole cube, we can assume that the preimage of each $m$-cube is a collection of $m$-cubes in $M$ and that $f$ maps each by a standard identification to the corresponding $m$-cell of $C$. Remove the interiors of these $m$-cubes and work in their complement. The map $f$ restricted to the boundary maps to the $(m-1)$-skeleton of $C$ and is transverse to centres of $(m-1)$-cells. Consider one such centre point $p$ in an $(m-1)$-cell $R$. By relative transversality we can assume that the preimage of $p$ is a 1-manifold with normal bundle mapped to a neighbourhood of $p$ in $R$. By expanding a small neighbourhood of $p$ onto the $(m-1)$-cell we can assume that the normal bundle is fibred by copies of $R$ mapped by the identity to $R$. We now excise the interiors of these bundles and we are mapping to the $(m-2)$-skeleton and we repeat the construction using the centres of $(m-2)$-cells. Continue using the centres of $(m-p)$-cells, for $p=3, \ldots, m$. The end result of this process is the required transverse map. Let $Q$ be the self-transverse immersed submanifold which comprises all the codimension 1 central subcubes in all the fibres (the cubes of $J^{1}(R)$ as $R$ varies). For example these would be the planes with bold borders in Figure 3). The cube bundles of varying dimensions that have been constructed form the required neighbourhood system of $Q$.

REMARK. We can describe $\widehat{\rho} / I^{n}$ as an explicit subbundle of the infinite trivial bundle in a similar way to the analogous construction in $[\mathbf{2 3}]$. To be precise we can embed $\hat{\rho}^{n}$ as a subbundle of $I^{n} \times \mathbb{R}^{n} \times \mathbb{R}^{n}$ as follows. Choose a small neighbourhood system for the image of $J^{1}\left(I^{n}\right)$ in $I^{n}$, regarded as usual as made of (trivial) cube bundles of varying dimensions with fibres which we shall call 'small' cubes (to distinguish them from the bigger cubes which comprise $I^{n}$ and $J^{1}\left(I^{n}\right)$ ). Without loss of generality assume that the function $v$ is constant on the preimage of each small cube. Think of the neighbourhood system as covered by double collars on the $I_{(i)}^{n}$ where each collar line lies in a small cube parallel to an edge. 
Now over each such line (corresponding to $p_{i} \in I_{(i)}^{n}$ say) turn the vector $v_{i}$ over in the plane determined by $v_{i}$ and $\bar{v}_{i}$ (which is the copy of $v_{i}$ in the other copy of $\mathbb{R}^{n}$ ); in other words make an explicit Möbius bundle in this plane over the line. Where vectors in two or more such bundles lie over the same point of $I^{n}$ they are contained in perpendicular planes, so there is no interference. The construction of $\widehat{\rho} /|X|$, where $X$ is a $\square$-space, now yields $\widehat{\rho}$ as an explicit subbundle of the infinite trivial bundle.

\section{C-manifolds}

Now suppose we are given a transverse map $f: M \rightarrow C$ where $C$ is a $\square$-set. Thus we have a self-transverse immersed submanifold $Q$ in $M$ and an identification of $M$ with the realisation of a $\square$-space structure $X$ on a neighbourhood system of $Q$. We say that the stable normal bundle $\nu / M$ of $M$ is twisted by $Q$ if it is identified with $\widehat{\rho} /|X|$.

We can now define a $C$-manifold. This is a manifold $M$ and a transverse map $f: M \rightarrow C$, such that the stable normal bundle $\nu / M$ of $M$ is twisted by the corresponding self-transverse immersed submanifold. There is an obvious concept of $C$-cobordism between $C$-manifolds and then we can define the $C$-bordism groups of a space $X$ by mapping $C$-manifolds and cobordisms into $X$ in the usual way. There is a dual concept of $C$-cobordism given by considering mock bundles with fibres $C$-manifolds.

5.3. TheOREM. The theory of $C$-bordism given by $C$-manifolds coincides with the theory $M C_{*}$ defined by $M C$. Similarly the two cobordism theories coincide.

Before proving the theorem we shall recall another theory considered by Mahowald [19] for which a geometric description is given in [23].

\section{The Mahowald spectrum}

The spectrum $M A$ (another of the spectra defined by Mahowald [19]) is pulled back from $M O$ by $\Omega^{2} \nu$ where $\nu: S^{3} \rightarrow B^{3} O$ is a generator. Mahowald [19] and Priddy [20] have proved that the composition $M A \rightarrow M O \rightarrow H \mathbb{Z}_{2}$ is an equivalence.

\subsection{Proposition. The map $M C \rightarrow M O$ factors as $M C \rightarrow M A \rightarrow M O$.}

We shall prove the proposition and the theorem together. We shall need to recall the geometric description of $M A_{*}(-)$ given in $[\mathbf{2 3}]$. We shall translate the language of [23] into the language of this paper. Roughly speaking, 'good position' as in $[\mathbf{2 3}]$ corresponds to the neighbourhood systems used here.

Consider a framed self-transverse immersion of codimension $1, Q \rightarrow M$. A neighbourhood system of $Q$ can be constructed as described above. Although this does not necessarily give $M$ a $\square$-space structure, twisting by $Q$ can be described in a very similar way. The details can be obtained from [23].

An $A$-manifold is a manifold $M$ with a framed self-transverse immersion of codimension 1, $f: Q \rightarrow M$, covered by an embedding $\widetilde{f}: Q \rightarrow M \times \mathbb{R}^{2}$ and such that $\nu_{M}$ is twisted by $Q$. The following result is proved in [23]. 
5.5. Theorem. The bordism theory determined by A-manifolds is the theory $M A_{*}$. Similarly the cohomology theories coincide.

\section{Proofs of Theorem 5.3 and Proposition 5.4}

An element of the homology group $M C_{m}(Y)$ is determined by a closed $m$-manifold $M^{m}$ together with a map $f: M \rightarrow Y$ and an isomorphism $F: \nu_{M} \rightarrow \phi_{C}^{*} \gamma$ where $\nu_{M}$ is the stable normal bundle of $M$ and $\gamma$ is the universal bundle over $B O$. Now $F$ restricts to a map $M \rightarrow|C|$ which may be assumed to be transverse by Theorem 5.2 and determines a codimension 1 self-transverse immersed submanifold $Q$ say.

It remains to prove that the isomorphism $F: \nu_{M} \rightarrow \phi_{C}^{*} \gamma$ is equivalent to $\nu_{M}$ being twisted by the diagram. To see this we shall use the spectrum $M A$ and the geometric description given above.

There is a well-known configuration space model $C_{2}\left(S^{1}\right)$ for $\Omega^{2} S^{3}$ :

$$
C_{2}\left(S^{1}\right)=\left(\coprod C_{2, k} \times_{\Sigma_{k}} I^{k}\right) / \sim
$$

where $C_{2, k}$ denotes ordered subsets of $\mathbb{R}^{2}$ containing $k$ points and

$$
\left[x_{1}, \ldots, x_{k}, t_{1}, \ldots, t_{k}\right] \sim\left[x_{1}, \ldots, \widehat{x}_{i}, \ldots, x_{k}, t_{1}, \ldots, \widehat{t}_{i}, \ldots, t_{k}\right] \quad \text { if } t_{i} \in\{0,1\} .
$$

The space of unordered sets $C_{2, k} / \Sigma_{k}$ can be identified with the classifying space of the braid group $\mathrm{Bbr}_{k}$. There is a filtration of $C_{2}\left(S^{1}\right)$ :

$$
* \subset F_{1} C_{2}\left(S^{1}\right) \subset \ldots \subset F_{k} C_{2}\left(S^{1}\right) \ldots C_{2}\left(S^{1}\right) \simeq \Omega^{2} S^{3},
$$

where $F_{k}$ is obtained from $F_{k-1}$ by attaching the bundle of cubes

$$
C_{2, k} \times_{\Sigma_{k}} I^{k} \longrightarrow \mathrm{Bbr}_{k}
$$

along its sphere bundle $C_{2, k} \times_{\Sigma_{k}} \partial\left(I^{k}\right) \rightarrow \mathrm{Bbr}_{k}$.

There is a similar model for $\Omega S^{2}$. Simply replace $C_{2, k}$ by $C_{1, k}$. There are maps $\phi_{k}: I^{k} \rightarrow C_{1, k} \times_{\Sigma_{k}} I^{k}$ given by $\left(t_{1}, \ldots, t_{k}\right) \mapsto\left[(0,0), \ldots,(k, 0), t_{1}, \ldots, t_{k}\right]$. These maps combine to give a homotopy equivalence $|T| \rightarrow C_{1}\left(S^{1}\right)$. The composition

$$
|T| \longrightarrow C_{1}\left(S^{1}\right) \subset C_{2}\left(S^{1}\right) \simeq \Omega^{2} S^{3} \stackrel{\Omega^{2} \nu}{\longrightarrow} B O
$$

is our standard map $s:|T| \rightarrow B O$, and so we have

$$
M C \longrightarrow M A \longrightarrow M O \longrightarrow H \mathbb{Z}_{2}
$$

and it follows that the map $M \rightarrow B O$ factors via $M A$.

The required result now follows from Theorem 5.5 above after noting that the descriptions of twisting given above and in $[\mathbf{2 3}]$ are effectively the same.

REMARK. The maps $\mathbb{S} \rightarrow M C$ (when $C$ is non-empty) and $M C \rightarrow M A \simeq H \mathbb{Z}_{2}$ can now be described geometrically. To get from $\mathbb{S}_{*}(-)$ to $M C_{*}(-)$ simply take the map to $C$ to be the constant map to a chosen vertex. To pass from $M C_{*}(-)$ to $M A_{*}(-)$ forget the map to $C$, remember only the codimension 1 self-transverse submanifold and allow normal bundles to be classified by the cube bundles over $\mathrm{Bbr}_{k}$. 


\section{Restrictions on normal bundles}

The manifolds which can occur in the definition of $M C_{n}(X)$ are restricted by the condition on the normal bundles. In particular we have the following result.

5.6. Proposition. If $M$ is an $n$-dimensional $C$-manifold then $\mathrm{wu}(M)=w(M)$, and $w_{i}(M)=0$ for $2 i>n$.

Proof. Recall that $\mathrm{wu}(M)=\mathrm{wu}(\nu(M))$ and $w(M)=w(\tau(M))$. The result now follows from the remark after Corollary 4.6 and the fact that $\mathrm{Wu}$ classes of manifolds vanish above half dimension.

\section{Rack and link homology theories}

In the case when $C=B X$ where $X$ is a rack, the geometric description of $M C_{*}$ can be simplified. A transverse map $f: M \rightarrow B X$ is equivalent to a framed codimension 2 submanifold of $M \times \mathbb{R}$ together with a representation of the fundamental rack in $X$ (see [12]). If $X$ is the fundamental rack of a link $L$, then we can regard the resulting theory as $L$-bordism, and this provides many more new invariants of links by calculating the values of this theory on test spaces.

There is also a 'truncated theory' given by using only the 2-skeleton of the rack space and this also makes good sense for any trunk as well. The resulting spectrum can be identified with $\mathbb{S} \wedge X$ where $X$ is the Thom complex of a plane bundle over the 2-skeleton.

\section{Operations}

The geometric description of $C$-manifolds given above allows us to define operations on these theories, which cover conjugate Steenrod square operations in $\mathbb{Z}_{2}$-cohomology.

5.7. Theorem. Let $C$ be a $\square$-set. There is an operation $M C_{n} \rightarrow M D_{n-k}$ where $D=J^{k}(C)$ and a similar operation $M C^{n} \rightarrow M D^{n+k}$. These operations correspond to a map of spectra $M C \rightarrow \Sigma^{k} M J^{k}(C)$.

In case $C=T$, the trivial $\square$-set, we can compose with $J^{k}(T) \rightarrow T$ to get a map $M T \rightarrow \Sigma^{k} M T$ which fits into the commutative diagram

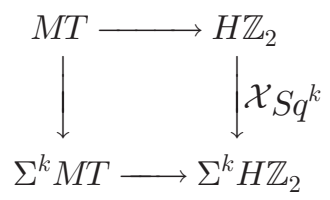

where the horizontal maps are the map $M T \rightarrow M A \cong H \mathbb{Z}_{2}$ considered above and its $k$-fold suspension.

Proof. We need a description of the $k$-tuple points of the immersion $Q$ determined by a transverse map $f: M \rightarrow|C|$ given in $[\mathbf{1 2}, \S 2]$, namely that there is an induced transverse map $f^{(k)}: Q^{(k)} \rightarrow J^{k}(C)$ where $Q^{(k)}$ is the manifold covering the $k$-tuple points of $Q$. It can be seen that the condition that the normal bundle is twisted by the immersion is inherited by the induced immersion in $Q^{(k)}$ and this defines the operations. 
Now the map $M T \rightarrow M A \cong H \mathbb{Z}_{2}$ is induced by the universal Thom class of $M T$ and the commutativity of the diagram then follows from the results of $[\mathbf{2 3}]$; see in particular Remark 3.6.

REMARK. The operation $M T \rightarrow \Sigma^{k} M T$ defined by Theorem 5.7 coincides with one of the operations defined by Mahowald on page 552 of [19].

\section{References}

1. R. Antolini, 'Cubical structures and homotopy theory', $\mathrm{PhD}$ Thesis, University of Warwick, 1996.

2. R. Antolini and B. Wiest, 'The singular cubical set of a topological space', Math. Proc. Cambridge Philos. Soc. 126 (1999) 149-154.

3. D. Bar-Natan, 'On the Vassiliev knot invariants', Topology 34 (1995) 423-472.

4. H. BAuEs, Commutator calculus and groups of homotopy classes, London Mathematical Society Lecture Note Series 50 (Cambridge University Press, 1981).

5. R. H. Bruck, A survey of binary systems (Springer, Berlin, 1958).

6. S. Buoncristiano, C. Rourke and B. Sanderson, A geometric approach to homology theory, London Mathematical Society Lecture Note Series 18 (Cambridge University Press, 1976).

7. W. Dreckmann, 'Distributivgesetze in der Homotopietheorie', PhD Thesis, Bonn, 1992.

8. R. FEnN, 'Historical background', http://www.maths.sussex.ac.uk//Staff/RAF/Maths/.

9. R. FEnN and C. Rourke, 'Racks and links in codimension two', J. Knot Theory Ramifications 1 (1992) 343-406.

10. R. Fenn, C. Rourke and B. Sanderson, 'Trunks and classifying spaces', Appl. Categ. Structures 3 (1995) 321-356.

11. R. Fenn, C. Rourke and B. Sanderson, 'James bundles and applications', Preprint, 1996, http://www.maths.warwick.ac.uk/ cpr/ftp/james.ps.

12. R. Fenn, C. Rourke and B. SAnderson, 'The rack space', Preprint, 2003, arXiv: math.GT/0304228.

13. R. Fenn, C. Rourke and B. Sanderson, 'A classification of classical links', Preprint, University of Warwick, 2004.

14. I. JAMES, 'Reduced product spaces', Ann. of Math. 62 (1955) 170-197.

15. I. James, 'On the suspension triad', Ann. of Math. 63 (1956) 191-247.

16. D. Joyce, 'A classifying invariant of knots; the knot quandle', J. Pure Appl. Algebra 23 (1982) 37-65.

17. U. Koschorke and B. Sanderson, 'Self-intersections and higher Hopf invariants', Topology 17 (1978) 283-290.

18. R. Lashof and S. Smale, 'Self-intersections of immersed manifolds', J. Math. Mech. 8 (1959) 143-157.

19. M. Mahowald, 'Ring spectra which are Thom complexes', Duke Math. J. 46 (1979) $549-559$.

20. S. Priddy, ' $K(Z / 2)$ as a Thom spectrum', Proc. Amer. Math. Soc. 70 (1978) 207-208.

21. D. Puppe, 'Some well known weak homotopy equivalences are genuine homotopy equivalences', INDAM, Rome, 1969/70, Symposia Mathematica V (Academic Press, London, 1971) 363-374.

22. C. Rourke and B. Sanderson, ' $\Delta$-sets, I: homotopy theory', Quart. J. Math. Oxford Ser. (2) 22 (1971) $321-338$. ' $\Delta$-sets, II: block bundles and block fibrations', ibid. 22 (1971) $465-485$.

23. B. SAnderson, 'The geometry of Mahowald orientations', Algebraic topology, Aarhus, 1978 (ed. J. L. Dupont and I. H. Madsen), Lecture Notes in Mathematics 763 (Springer, Berlin, 1979) $152-174$.

24. V. A. VAssiliev, 'Cohomology of knot spaces', Theory of singularities and its applications (ed. V. I. Arnol'd), Advances in Soviet Mathematics 1 (American Mathematical Society, Providence, RI, 1990) 23-69.

25. B. Wiest, 'Knots, links, and cubical sets', PhD Thesis, University of Warwick, 1997.

Roger Fenn

Department of Mathematics

University of Sussex

Falmer

Brighton BN1 9QH

United Kingdom

R.A.Fenn@sussex.ac.uk
Colin Rourke and Brian Sanderson

Mathematics Institute

University of Warwick

Coventry CV4 7AL

United Kingdom

cpr@maths.warwick.ac.uk

bjs@maths.warwick.ac.uk 